\title{
DISTRIBUCIÓN GEOGRÁFICA Y CONSERVACIÓN DE NUEVE ESPECIES DEL GÉNERO Ferocactus (CACTACEAE) EN MÉXICO
}

\section{GEOGRAPHIC DISTRIBUTION AND CONSERVATION OF NINE SPECIES OF THE GENUS Ferocactus (CACTACEAE) IN MEXICO}

\author{
Claudia Ballesteros-Barrera*, Osiris Aguilar-Romero, \\ Rocío Zarate-Hernández y Lorenzo Ballesteros-Tapia
}

Departamento de Biología, Unidad Iztapalapa, Universidad Autónoma Metropolitana. Av. San Rafael Atlixco 186, Col. Vicentina. 09340, México, D.F.

*Autor para correspondencia (bbc7@xanum.uam.mx)

\section{RESUMEN}

El género Ferocactus presenta una taxonomía, historia filogenética y evolutiva muy compleja, donde el aislamiento geográfico y reproductivo han sido importantes para su diversificación y evolución. Sin embargo, es alarmante el número de especies que se encuentran en alguna categoría de riesgo. Por tanto, es importante conocer la distribución de cada una de las especies, entender las causas ambientales que determinan su distribución, así como la pérdida de su hábitat debido al cambio de uso de suelo. En este trabajo se usaron los modelos de nicho ecológico para estimar la distribución potencial de nueve especies de Ferocactus sujetas a protección según la legislación mexicana. Por medio de un Sistema de Información Geográfica se obtuvieron los perfiles bioclimáticos de cada especie, así como la influencia de las variables climáticas en la distribución. Asimismo, se evaluó el efecto del cambio de uso de suelo en su área de distribución y se calculó el área resguardada dentro de alguna Área Natural Protegida. Los resultados mostraron que el hábitat perdido es mayor en las especies que se encuentran en el centro de México en comparación con las que habitan en la península de Baja California, y que la representatividad de la mayoría de las especies en Áreas Naturales Protegidas es baja. Por ello, sería importante analizar la posibilidad de re-evaluar las categorías de riesgo de las especies, reunir esfuerzos en la identificación de áreas potenciales adecuadas para considerarse en los planes o estrategias de conservación o recuperación, y sugerir el decreto de nuevas áreas (o la extensión de éstas) que protejan el hábitat de las especies.

Palabras clave: Ferocactus, cactáceas, distribución potencial, conservación, nicho climático, categorías de riesgo de extinción.

\section{SUMMARY}

The genus Ferocactus has complex taxonomy and phylogenetic and evolutionary history, affected greatly by geographic and reproductive isolation in its diversification and evolution. However, the number of species classified in risk categories is shocking, therefore the knowledge on species' distribution patterns and their environmental determinants, as well as the processes of habitat loss due to land use change is important. In this paper, we used ecological niche modeling to generate potential distribution of nine species of Ferocactus protected by Mexican law, then using geographic information systems we obtained the bioclimatic profile of each species and the influence of climatic variables in their geographic distribution. We also evaluated the effects of the change of land use in their distribution area and estimated the safeguarded area within a Natural Protected Area. The results show that habitat loss is greater in the species whose distribution area is in the center of México compared to those living in the Baja California Peninsula and the representativeness of most species is low in Natural Protected Areas. It might be necessary to re-evaluate the risk categories of species, as well as coordinate efforts to identify potential areas suitable for conservation strategies or recovery plans and to promote the decree of new areas (or extension thereof) to protect the habitat of the species.

Index words: Ferocactus, cacti, potential distribution, conservation, climatic niche, extinction risk categories.

\section{INTRODUCCIÓN}

La familia Cactaceae es originaria de América, se distribuye principalmente en las regiones áridas y semiáridas (Anderson, 2001), y existen alrededor de 1900 especies en el mundo, comprendidas en 125 géneros (Jiménez-Sierra, 2011). México es considerado el más importante centro de evolución y diversificación de esta familia, con alrededor de 68 géneros y 669 especies, de las cuales 518 (77\%) son exclusivas del país, por lo que posee un elevado índice de endemismos (Cota-Sánchez, 2008; Guzmán et al., 2007). Desafortunadamente una alta proporción de especies presenta problemas de conservación debido a sus características biológicas como son: la baja tasa de crecimiento y reclutamiento, la baja supervivencia de las semillas y plántulas, áreas de distribución restringidas y especialización a condiciones edáficas (Hernández y Godínez, 1994).

Otro aspecto restrictivo importante lo constituyen las actividades humanas, como el cambio en el uso de suelo, la introducción de especies exóticas, la extracción directa de plantas con fines alimenticios o bien con fines de ornato (Arias et al., 2005, Jiménez-Sierra, 2011) ya que cerca de $86 \%$ de las especies amenazadas que se usan en horticultura son extraídas de poblaciones silvestres (Goettsch et al., 2015). Debido a estos factores las cactáceas ocupan 
los primeros lugares en los listados publicados por instancias nacionales e internacionales en donde se determina el estatus de conservación de las especies, como la Norma Oficial Mexicana NOM-059-SEMARNAT-2010 (DOF, 2010); la Convención sobre Comercio Internacional de Especies Amenazadas de Fauna y Flora Silvestres (CITES, 2017) y la Lista Roja de la Unión Mundial para la Naturaleza (IUCN, 2016).

De los 275 taxones de cactáceas que incluye la NOM059-SEMARNAT-2010, diez corresponden al género Ferocactus. Estas cactáceas conocidas como "cactus de barril" o "biznagas", son vistosas por su forma globular y por el color de las flores. Se distribuyen en las zonas cálido secas del suroeste de Estados Unidos de América y en la región norte de México (Arias et al., 2012). Los estudios filogenéticos que se han realizado concluyen que Ferocactus es un grupo polifilético, que incluye a los géneros Ferocactus, Glandulicactus, Leuchtenbergia, Stenocactus y Thelocactus (Vázquez-Sánchez et al., 2013).

De acuerdo con la NOM-059-SEMARNAT-2010, uno de los criterios que se utilizan para designar en qué categoría de riesgo se encuentran los taxones es la extensión de la distribución con relación a la extensión territorial del país (DOF, 2010). Sin embargo, es poco el conocimiento que existe referente a muchas especies mexicanas en lo que respecta al área de distribución geográfica, el tamaño de las poblaciones, las causas ambientales que determinan su distribución, así como el estado actual del hábitat del taxón debido al impacto de las actividades humanas (Contreras-Medina et al., 2010). Adicionalmente, se ha reconocido la importancia de saber la proporción del área de distribución que ocupan las especies, con respecto a la superficie total de las Áreas Naturales Protegidas (ANP), ya que éstas constituyen una de las principales estrategias de conservación, por lo que es pertinente que se representen adecuadamente la biodiversidad de una región de manera que garanticen su persistencia (Londoño-Murcia y Sánchez-Cordero, 2011).

Recientemente se ha desarrollado el modelado de nicho ecológico (MNE) como método para generar hipótesis sobre la distribución potencial de las especies (Peterson et al., 2002); el cual se basa en el concepto de Nicho Ecológico de Hutchinson (1957). Los MNE se han utilizado en la búsqueda de patrones de distribución de las especies (Ferrier y Guisan, 2006), exploraciones biológicas dirigidas (Raxworthy et al., 2003), y en la biología de la conservación (Sánchez-Cordero et al., 2005), entre muchos otros ejemplos.

El objetivo de este trabajo fue reportar para nueve especies de Ferocactus sujetas a protección según la legislación ambiental federal mexicana NOM-059-SEMARNAT-2010, las siguientes variables: la distribución actual conocida y la distribución potencial, el área de su distribución que aún no ha sido modificada por cambio de uso de suelo, así como la representatividad de estas especies amenazadas en las áreas naturales protegidas (ANP) y presentar los perfiles bioclimáticos para cada especie.

\section{MATERIALES Y MÉTODOS}

Para generar los modelos de nicho ecológico (MNE) se construyó una base de datos de las localidades de registro de nueve especies del género Ferocactus que se encuentran dentro de alguna categoría de riesgo de acuerdo con la Norma Oficial Mexicana NOM-059-SEMARNAT-2010 (DOF, 2010). Las especies que se encuentran sujeta a protección especial (Pr) son F. cylindraceus, F. haematacanthus, F. histrix, F. johnstonianus y F. pilosus; mientras que F. rectispinus, F. townsendianus, F. viridescens y F. chrysacanthus se encuentran clasificadas como amenazada (A). Esta información se obtuvo de los portales digitales mundiales y nacionales, como Global Biodiversity Information Facility (GBIF; http://www.gbif.org/), de la Red Mundial de Información sobre Biodiversidad (REMIB; http://www.conabio. gob.mx/remib/doctos/remib_esp.html) y la Unidad de Información para la Biodiversidad (UNIBIO; http://unibio. unam.mx/), además de literatura científica y especializada (Pilbeam y Bowdery, 2005).

A través de un Sistema de Información Geográfica (SIG), las coordenadas geográficas fueron revisadas, verificadas y corregidas de ser necesario; además estas localidades fueron comparadas con los mapas de distribución conocida de las especies que se encuentran en el catálogo de metadatos geográficos de la Comisión Nacional para el Uso y Conocimiento de la Biodiversidad (CONABIO; http:// www.conabio.gob.mx/informacion/gis/). Además, se emplearon coberturas digitales a una resolución de $1 \mathrm{~km}^{2}$ de 19 variables ambientales relacionadas con valores de temperatura y precipitación generadas para México (CuervoRobayo et al., 2013), además de cuatro variables topográficas (U.S. Geological Survey Hydro 1K http://usgs.gov) (Cuadro 1).

Se utilizó el algoritmo MaxEnt (Phillips et al., 2006) reconocido como uno de los más robustos para realizar los MNE, porque puede ajustar los modelos a partir un número reducido de datos; además, requiere únicamente información sobre la presencia de la especie, y ha mostrado un buen desempeño en una amplia variedad de especies y regiones (Elith et al., 2006). La explicación detallada sobre el funcionamiento de MaxEnt, se describe en diversos artículos, principalmente por Phillips et al. (2006). Para la validación estadística de los modelos, el programa proporciona el 
Cuadro1. Coberturas digitales utilizadas en el modelo de distribución geográfica potencial de las especies de Ferocactus.

\begin{tabular}{ll}
\hline Clave & Variables ambientales \\
\hline Bio 1 & Temperatura media anual $\left({ }^{\circ} \mathrm{C}\right)$. \\
Bio 2 & Oscilación diurna de la temperatura $\left({ }^{\circ} \mathrm{C}\right)$. \\
Bio 3 & Isotermalidad $\left({ }^{\circ} \mathrm{C}\right)$. \\
Bio 4 & Temperatura estacional (coeficiente de variación) $(\%)$. \\
Bio 5 & Temperatura máxima del mes más cálido $\left({ }^{\circ} \mathrm{C}\right)$. \\
Bio 6 & Temperatura mínima del mes más frío $\left({ }^{\circ} \mathrm{C}\right)$. \\
Bio 7 & Oscilación anual de la temperatura $\left({ }^{\circ} \mathrm{C}\right)($ cociente entre parámetros 5 y 6$)$. \\
Bio 8 & Temperatura media del trimestre más húmedo $\left({ }^{\circ} \mathrm{C}\right)$. \\
Bio 9 & Temperatura media del trimestre más seco $\left({ }^{\circ} \mathrm{C}\right)$. \\
Bio 10 & Temperatura media del trimestre más cálido $\left({ }^{\circ} \mathrm{C}\right)$. \\
Bio 11 & Temperatura media del trimestre más frío $\left({ }^{\circ} \mathrm{C}\right)$. \\
Bio 12 & Precipitación anual (mm). \\
Bio 13 & Precipitación del mes más húmedo $(\mathrm{mm})$. \\
Bio 14 & Precipitación del mes más seco $(\mathrm{mm})$. \\
Bio 15 & Precipitación estacional (coeficiente de variación) $(\%)$. \\
Bio 16 & Precipitación del trimestre más húmedo $(\mathrm{mm})$. \\
Bio 17 & Precipitación del trimestre más seco $(\mathrm{mm})$. \\
Bio 18 & Precipitación del trimestre más cálido $(\mathrm{mm})$. \\
Bio 19 & Precipitación del trimestre más frío $(\mathrm{mm})$. \\
& Variables topográficas \\
\hline & Aspecto \\
\hline & Elevación (m) \\
& Orientación de la pendiente \\
\hline
\end{tabular}

valor de la curva ROC (Receiver Operating Characteristic) y el estadístico AUC (Area Under the ROC Curve), el cual muestra la bondad de los modelos con valores que pueden estar entre 0 y 1 , donde un valor de 0.5 significa que el modelo presenta una clasificación al azar, y un valor de 1 indica que el modelo ha separado presencias de ausencias con un ajuste perfecto (Felicísimo et al., 2011).

Una vez obtenidos los MNE para cada especie, por medio de un SIG se transformaron a mapas digitales binarios (presencia/ausencia), donde un valor igual a 1 refiere una posible presencia (hábitat idóneo) mientras que un valor de 0 es igual a ausencia (hábitat no idóneo). Se consideró como punto de corte el valor del décimo percentil, para maximizar la sensibilidad y minimizar la especificidad (Cruz-Cárdenas et al., 2014), lo que muestra solo los pixeles que son iguales o mayores al valor establecido.

A los mapas binarios de cada una de las especies se les eliminaron las áreas que representan un hábitat perturba- do para la presencia de los Ferocactus; es decir, aquellos polígonos referentes a las zonas urbanas, agrícolas, desprovistas de vegetación, cuerpos de agua, zonas ganaderas, entre otras, y se utilizó como referencia el Inventario Nacional Forestal de México (Serie V, Escala 1:250 000; INEGI, 2013). Con ello se estimó el área perdida (en términos de porcentaje) dada por el cambio del uso del suelo, y se identificaron las áreas que actualmente presentan algún estado de conservación o hábitat apropiado para las especies. Finalmente, se intersectaron con los polígonos de las Áreas Naturales Protegidas de México (ANP; CONANP, 2012), y se calculó el porcentaje de área de presencia de cada especie que se encuentra resguardada dentro de alguna de las ANP.

Para estimar los perfiles bioclimáticos de cada especie se utilizó la extensión de BIOCLIM (BIOCLIMav Beta 1.2) compatible con el SIG, para extraer los valores de las coberturas digitales (Cuadro 1) para cada registro de presencia de las especies; con los datos obtenidos se 
calcularon los valores promedio de cada variable climática. Adicionalmente, con los datos de los perfiles se realizó un análisis de función discriminante (AFD) mediante el programa Statistica 10 (Statsoft v. 2010), con el fin de evaluar si existen diferencias significativas entre los nichos ambientales de las nueve especies de Ferocactus, en función de los factores climáticos considerados como variables discriminatorias y como variable clasificatoria a las mismas especies.

\section{RESULTADOS Y DISCUSIÓN}

Se obtuvo un total de 1092 datos de presencia, los cuales varían entre las especies, con un mínimo de 16 registros como en el caso de F. johnstonianus, y hasta 247 para F. pilosus (Cuadro 2). El análisis de las curvas ROC para los MNE de todas las especies proporcionaron valores del área bajo la curva (AUC) mayores a 0.9 lo que indica que los modelos fueron estadísticamente mejores que al azar.

En la mayoría de los casos el área de distribución potencial predicha por los modelos fue mayor que la conocida por los registros, lo que se puede deber por una parte a que los modelos pueden estar sobre representando el área de distribución geográfica de una especie porque indican la idoneidad del hábitat para el desarrollo de poblaciones de una especie concreta, pero sin considerar otros factores como las interacciones biológicas (Ferrier y Guisan, 2006).

Otra explicación podría ser el síndrome del colector, que refiere a que se muestrean sitios cercanos a las carreteras o a ciertas localidades conocidas, lo que es una limitante en el conocimiento de la distribución de las especies. En este sentido, los modelos permiten llenar esos huecos del conocimiento cada vez con mayor confiabilidad (Soberón y Peterson, 2005), por lo que los sitios predichos en el modelo podrían servir de guía para dirigir el trabajo de campo hacia sitios específicos para verificar si se encuentra la especie (Villaseñor y Téllez-Valdés, 2004).

Los resultados obtenidos a partir de los MNE, el cambio de uso de suelo y las ANP por especie fueron los siguientes (Cuadros 2 y 3 , Figuras 1 A y B):

F. chrysacanthus. Esta especie presenta registros en las islas Cedros y San Benito en Baja California, así como en Baja California Sur. La distribución potencial predicha es aproximadamente de $5211 \mathrm{~km}^{2}$ y se ajusta a estos estados. Se calcula que $2.2 \%$ de esta área ha sido modificada por algún tipo de cambio de uso de suelo; además, muestra un alto porcentaje de su área de distribución (94.6\%) dentro de la Reserva de la Biosfera (RB) El Vizcaíno. A pesar de que su área de distribución es restringida y que se clasifica como amenazada según la Norma Mexicana, en peligro según la IUCN y en el Apéndice II del CITES, sus principales amenazas siguen siendo el saqueo histórico inducido por la llamativa apariencia de los ejemplares, además de la modificación del hábitat en la isla debido a un asentamiento de varios cientos de personas que laboran para una empresa exportadora de sal (León de la Luz, 2003a).

F. cylindraceus. Debido a que tienen una distribución más amplia y a que no es endémica, se encuentra catalogada como sujeta a protección aspecial según la NOM-059-SEMARNAT-2010, de menor preocupación según la IUCN, y está en el Apéndice II del CITES. Se distribuye en Baja California y en una pequeña área de Sonora, y en los Estados Unidos de America en los estados de California, Nevada y

Cuadro 2. Número de registros de presencia en México de las especies de Ferocactus, categorías de riesgo, valores de extensión de la distribución geográfica potencial, pérdida de área por cambio de uso del suelo y áreas conservadas en las áreas naturales protegidas.

\begin{tabular}{llcccc}
\hline Especie & Categoría de riesgo & $\begin{array}{c}\text { Número de } \\
\text { registros }\end{array}$ & $\begin{array}{c}\text { Distribución } \\
\text { potencial }\left(\mathrm{km}^{2}\right)\end{array}$ & $\begin{array}{c}\text { Área perdida por } \\
\text { uso de suelo (\%) }\end{array}$ & $\begin{array}{c}\text { Área } \\
\text { protegida (\%) }\end{array}$ \\
\hline F. chrysacanthus & Amenazada & 24 & 5,211 & 2 & 95 \\
F. cylindraceus & Sujeta a protección especial & 56 & 27,411 & 7 & 20 \\
F. haematacanthus & Sujeta a protección especial & 33 & 8,264 & 44 & 13 \\
F. histrix & Sujeta a protección especial & 94 & 61,066 & 23 & 8 \\
F. johnstonianus & Sujeta a protección especial & 16 & 1,056 & 10 & 100 \\
F. pilosus & Sujeta a protección especial & 247 & 298,007 & 15 & 8 \\
F. rectispinus & Amenazada & 28 & 17,370 & 7 & 11 \\
F. townsendianus & Amenazada & 58 & 37,475 & 14 & 2 \\
F. viridescens & Amenazada & 52 & 12,983 & 30 & 1 \\
\hline
\end{tabular}


Cuadro 3. Perfiles bioclimáticos (valores promedio) de las especies del género Ferocactus.

\begin{tabular}{lcrrrrrrrr}
\hline Variables & \multicolumn{7}{c}{ Especies de Ferocactus } \\
\cline { 2 - 10 } ambientales & F.chr & F. cyl & F. hae & F. his & F. joh & F. pil & F. rec & F. tow & F. vir \\
\hline Bio 1 & 19.7 & 18.7 & 15.0 & 17.5 & 19.8 & 17.9 & 21.7 & 21.6 & 16.8 \\
Bio 2 & 11.0 & 16.4 & 14.4 & 15.8 & 14.3 & 15.8 & 15.8 & 15.0 & 11.9 \\
Bio 3 & 0.6 & 0.5 & 0.7 & 0.6 & 0.5 & 0.6 & 0.5 & 0.6 & 0.5 \\
Bio 4 & 1.0 & 2.3 & 0.6 & 0.9 & 1.8 & 1.1 & 1.7 & 1.4 & 1.2 \\
Bio 5 & 29.7 & 37.7 & 26.1 & 30.5 & 35.9 & 30.4 & 37.0 & 34.8 & 27.9 \\
Bio 6 & 10.2 & 2.6 & 4.5 & 4.8 & 6.6 & 4.9 & 7.4 & 8.2 & 5.9 \\
Bio 7 & 19.5 & 35.1 & 21.6 & 25.7 & 29.3 & 25.4 & 29.5 & 26.6 & 22.0 \\
Bio 8 & 16.5 & 13.6 & 16.2 & 19.6 & 13.8 & 20.8 & 28.1 & 25.9 & 13.0 \\
Bio 10 & 23.6 & 27.4 & 16.9 & 20.5 & 27.2 & 21.2 & 28.4 & 27.0 & 21.6 \\
Bio 11 & 16.4 & 10.8 & 12.8 & 13.7 & 13.7 & 13.6 & 15.5 & 16.7 & 12.7 \\
Bio 12 & 77.7 & 202.2 & 542.1 & 483.4 & 97.1 & 438.3 & 172.7 & 187.5 & 257.3 \\
Bio 13 & 4.2 & 9.2 & 28.8 & 24.6 & 4.6 & 18.5 & 10.6 & 16.4 & 12.6 \\
Bio 15 & 89.0 & 71.1 & 85.5 & 83.7 & 67.3 & 64.6 & 96.8 & 111.8 & 90.6 \\
Bio 16 & 45.5 & 101.6 & 270.0 & 263.0 & 42.7 & 194.2 & 103.4 & 128.2 & 148.1 \\
Bio 17 & 0 & 1.03 & 13.8 & 20.1 & 0 & 39.1 & 0 & 0 & 0 \\
Bio 18 & 9.29 & 34.81 & 156.0 & 194.6 & 27.44 & 181.8 & 95.14 & 108.0 & 10.6 \\
Bio 19 & 44.1 & 91.7 & 26.2 & 34.6 & 41.7 & 46.8 & 44.2 & 36.9 & 141.4 \\
\hline
\end{tabular}

F. chr: F. chrysacanthus; F. cyl: F. cylindraceus; F. hae: F. haematacanthus; F. his: F. histrix; F. joh: F. johnstonianus; F. pil: F. pilosus; F. rec: F. rectispinus; F. tow: F. townsendianus; F. vir: F. viridescens.

Arizona. En cuanto a su distribución potencial para México se calculó en $27,411 \mathrm{~km}^{2}$, donde $6.6 \%$ de esta superficie ha sido afectada por la pérdida de hábitat, mientras que un $19.5 \%$ se encuentra dentro del área de protección de flora y fauna (APFyF) en el Valle de los Cirios.

F. haematacanthus. Es una especie endémica que se encuentra como sujeta a protección especial según la NOM059-SEMARNAT-2010, en peligro según la IUCN y en el Apéndice II del CITES. Se ha registrado en el área oriental de Puebla, aunque el modelo de distribución predice un área total de $8264 \mathrm{~km}^{2}$ que incluye una porción del estado de Oaxaca. De su área de distribución $44.5 \%$ de su área de distribución presenta algún grado de perturbación y tan sólo 13.7 \% está dentro de la RB de Tehuacán-Cuicatlán. Se encuentran bajo amenaza debido a las actividades agrícolas, particularmente quemas y enfermedades, además de la actividad de los coleccionistas (Meza, 2011).

F. histrix. Es una especie endémica cuyas localidades se encuentran presentes en Durango, Zacatecas, Aguascalientes, Jalisco, San Luis Potosí, Guanajuato, Querétaro e Hidalgo (Pilbeam y Bowdery, 2005). Se encuentra en la categoría de sujeta a protección especial según la NOM059-SEMARNAT-2010, amenazada según la IUCN y en el Apéndice II del CITES. De los 61,066 km² del área de distri- bución predicha $23 \%$ muestran cambios en el uso de suelo y $7.7 \%$ se encuentran protegidos dentro de las RB Barranca de Metztitlán, Sierra Gorda y Sierra Gorda de Guanajuato. Las poblaciones de esta especie han sido saqueadas para procesarla y elaborar el dulce conocido como "acitrón" (Jiménez-Sierra, 2011).

F. johnstonianus. Esta especie endémica se encuentra como sujeta a protección especial según la NOM-059-SEMARNAT-2010, menor preocupación según la IUCN y en el Apéndice II del CITES. Es la especie que presenta la mayor protección o conservación, al mostrar toda su área de distribución $(1056$ km²) en la Isla Ángel de la Guarda que forma parte de la RB Zona Marina Bahía de los Ángeles (Baja California). Sin embargo, se identifica $10.2 \%$ con algún grado de perturbación antrópica, quizá debido a la cercanía de la Isla al poblado que se han incrementado los viajes turísticos a la isla, lo que puede provocar una alteración del hábitat (Delgadillo, 2004a).

F. pilosus. Los registros de esta especie endémica se encuentran en los estados de San Luis Potosí, Zacatecas, Durango, Nuevo León, Coahuila y Tamaulipas (Pilbeam y Bowdery, 2005). Se identificaron $298,007 \mathrm{~km}^{2}$ de distribución potencial, de los cuales $15.8 \%$ presentan algún cambio en uso del suelo, mientras que $7.8 \%$ se encuentra potencialmente en la RB de 

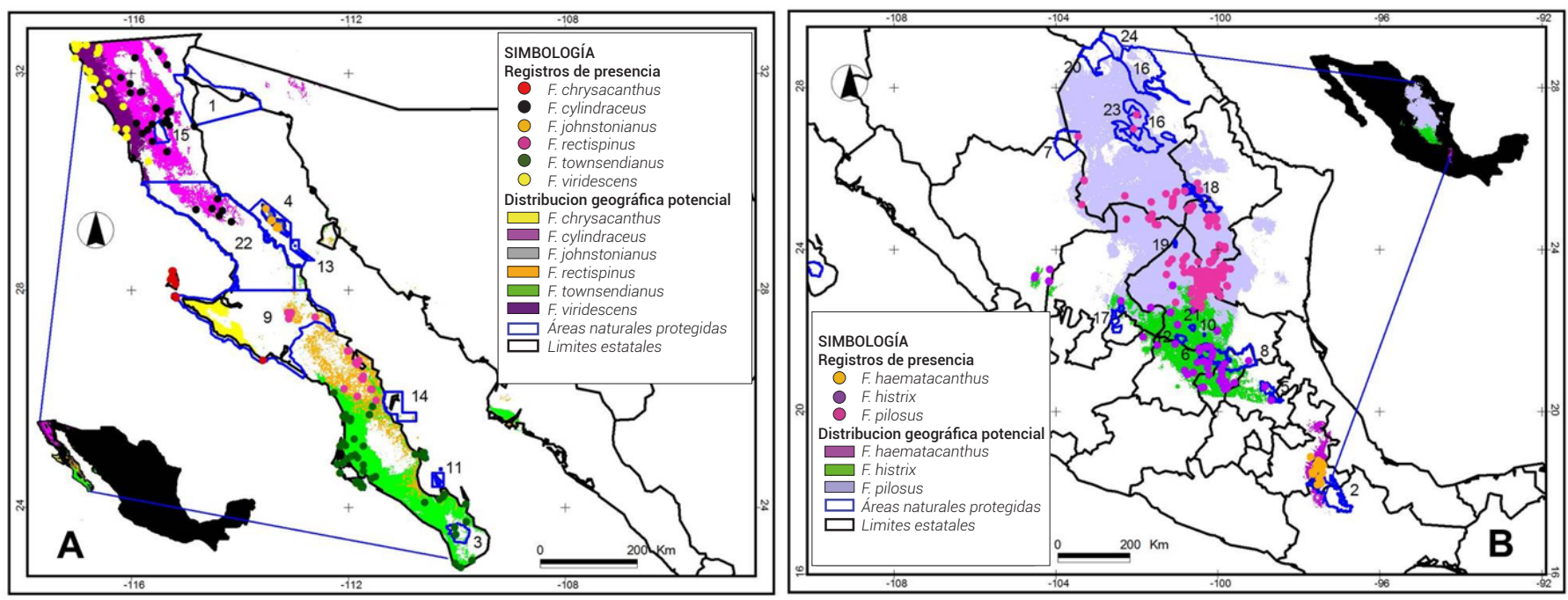

Figura 1. Mapa de las localidades de colecta y de la distribución geográfica potencial de especies del género Ferocactus, registros de presencia y su representatividad en las Áreas Naturales Protegidas. A) Especies localizadas en la Península de Baja California y B) Especies localizadas en el Altiplano y Centro de México. Las Reservas de la Biosfera están asignadas del número 1 al 9 (1: Alto Golfo de California y Delta del Río Colorado, 2: Tehuacán - Cuicatlán, 3: Sierra La Laguna, 4: Bahía de los Ángeles, Canales de Ballenas y Salsipuedes, 5: Barranca de Metztitlán, 6: Sierra Gorda de Guanajuato, 7: Mapimí, 8: Sierra Gorda y 9: El Vizcaíno), los Parques Nacionales del 10 al 15 (10: El Potosí, 11: Zona Marina del Archipiélago de Espíritu Santo, 12: Gogorrón, 13: Archipiélago de San Lorenzo, 14: Bahía de Loreto y 15: Sierra de San Pedro Mártir), las Áreas de Protección de los Recursos Naturales Zona Protectora Forestal del 16 al 18 (16: CADNR004, 17: CADNR001 y 18: CADNR026) y las Áreas de Protección de Flora y Fauna del 19 al 24 (19: Sierra La Mojonera, 20: Ocampo, 21: Sierra de Álvarez, 22: Valle de los Cirios, 23: Cuatrociénegas y 24: Maderas del Carmen).

Mapimí, en las APFyF de Cuatrociénegas, Ocampo y Maderas del Carmen, y el Área de Protección de los Recursos Naturales Zona Protectora Forestal (APRNZPF) CADNR004, aunque no hay registros de colecta. Se encuentra catalogada como sujeta a protección especial según la NOM-059-SEMARNAT-2010, Menor preocupación según la IUCN y en el Apéndice II del CITES. Los factores de riesgo que afectan a las poblaciones de la especie son el saqueo selectivo y la destrucción del hábitat por el sobrepastoreo, la deforestación y la construcción de autopistas y ampliación de carreteras (Meza, 2011).

F. rectispinus. Las localidades de colecta para esta especie endémica se encuentran en Baja California Sur. Se clasifica en la categoría de amenazada según la NOM-059-SEMARNAT-2010 y en el Apéndice II del CITES. El modelo de distribución mostró un área potencial de 17,370 km², de los cuales $7.4 \%$ presentan algún tipo de pérdida de hábitat y sólo $11 \%$ está dentro de la RB del Vizcaíno. Sus factores de riesgo son la destrucción del hábitat, el consumo de los individuos para el ganado y el saqueo hormiga por parte de coleccionistas de cactos (León de la Luz, 2003b).

F. townsendianus. Se encuentra en la categoría de amenazada según la NOM-059-SEMARNAT-2010 y en el Apéndi- ce II del CITES. Se le ha registrado en Baja California que incluyen las islas de Margarita y Magdalena, y en las islas Espíritu Santo, San Francisco y San José. De los 37,475 $\mathrm{km}^{2}$ de distribución geográfica potencial predicha por el modelo, $13.7 \%$ ha sido modificada por las actividades humanas y sólo $1.8 \%$ se encuentra albergado en la RB de Sierra Laguna. Las principales amenazas son la colecta indiscriminada, el uso como forraje para el ganado y la destrucción del hábitat para la implantación de zonas agrícolas (León de la Luz, 2003c).

F. viridescens. Esta especie no endémica presenta una pérdida de hábitat de $30 \%$ y aunque no hay registros de colecta en alguna ANP, potencialmente $0.7 \%$ de los 12 , $983 \mathrm{~km}^{2}$ identificados en Baja California podrían estar en la APFyF del Valle de los Cirios. Se ubica en la categoría de amenazada según la NOM-059-SEMARNAT-2010, como de Menor Preocupación por la IUCN y en el Apéndice II del CITES. Las poblaciones se encuentran con un alto grado de amenaza por las actividades humanas que se desarrollan en los centros de población, así como la urbanización de nuevos terrenos (Delgadillo, 2004b).

En general, los resultados obtenidos con respecto a la pérdida de área por cambio de uso de suelo y que por ende 
conlleva a la pérdida del hábitat de las especies, muestran una variación importante de 2 a 40 \% de la distribución potencial predicha. Las dos especies con mayor pérdida de área, F. haematacanthus y F. histrix, se encuentran distribuidas en la zona centro del país que es donde se encuentran las ciudades más pobladas, así como zonas de importante actividad humana como la agricultura y la industria; de la misma forma, en el área de distribución de $F$. viridescens se encuentran las ciudades más importantes y pobladas de la Península de Baja California, como Mexicali, Tijuana y Ensenada, donde se desarrolla la industria manufacturera, la hotelería y el turismo, así como la producción agrícola, ganadera y pesquera (INEGI, 2016).

Para el resto de las especies que habitan en la península de Baja California y en el Desierto Chihuahuense al parecer no se ha dado un cambio radical en el uso de suelo, de acuerdo con información del INEGI. La vegetación de las zonas áridas y semiáridas parecería la menos afectada en cuanto a su cobertura (una reducción de $17 \%$ de la original), pero esto puede ser reflejo de que su alteración (por ejemplo, por sobrepastoreo) no es tan fácil de cuantificar, por lo menos en estas escalas espaciales, por lo que es difícil distinguir el estado de conservación (vegetación primaria vs. vegetación secundaria) de estos ecosistemas (Challenger y Dirzo, 2009).

Esta problemática toma mayor relevancia al considerarse otros factores generados por las actividades humanas; tal es el caso de la extracción de individuos o colonias silvestres de algunas especies de cactáceas, cuyos usos están altamente relacionados con su valor económico o bien con fines alimenticios, lo cual aunado a la propia biología de éstas (metabolismo tipo CAM), hace que la recuperación sea muy lenta (Jiménez-Sierra, 2011).

Es de resaltar la baja incidencia de las especies de Ferocactus estudiadas dentro de las ANP del país, ya que salvo F. johnstonianus y F. chrysacanthus cuyas áreas están completamente inmersas en alguna ANP, el resto están desprotegidas. Más preocupante aún es el hecho de que $F$. townsendianus y F. viridescens consideradas en categoría de amenazada o el caso de F. histrix, cuyas áreas de distribución están siendo impactadas por cuestiones antropogénicas y sus áreas de distribución no se encuentran protegidas en alguna ANP. Con base en lo anterior, se considera analizar con mayor detalle este tema a fin de proponer nuevas ANP y de proteger a un mayor número de especies.

En cuanto a las condiciones climáticas en las que se encuentran las especies, se revisó el intervalo ambiental en el que se distribuyen las colectas de cada especie por medio de los perfiles bioclimáticos (Cuadro 3). Con ello se aporta información nueva que permite identificar los intervalos fisiológicos de las especies, así como los sitios con las condiciones ambientales donde pueden establecerse o permanecer las especies, se pueden realizar inferencias sobre sus tolerancias o requerimientos ambientales, lo que a su vez permite inferir en cierto modo las tolerancias fisiológicas de las especies.

Al graficar los valores de la temperatura media anual (Bio1) contra la precipitación anual (Bio12) (Figura 2), se puede observar un acomodo ambiental y geográfico de las especies analizadas con una tendencia de mayor precipitación y menor temperatura con $F$. haematacanthus (temperatura de $15{ }^{\circ} \mathrm{C}$ y precipitación de $542.1 \mathrm{~mm}$ ), en contraste con F. chrysacanthus y F. johnstonianus con las menores precipitaciones $(77.7 \mathrm{~mm}$ y $97.1 \mathrm{~mm}$, respectivamente), y con $F$. townsendianus y $F$. rectispinus con las mayores temperaturas $\left(21.6^{\circ} \mathrm{C}\right.$ y $21.7^{\circ} \mathrm{C}$, respectivamente), los cuales se ubican desde el centro al sur de Baja California Sur.

Sin embargo, al analizar los perfiles bioclimáticos desde un punto de vista multivariado por medio de un análisis de función discriminante (AFD), se forman grupos de especies asociados con climas similares, con lo cual se estableció la importancia de las variables. Los resultados indican que los valores de las dos primeras funciones discriminantes son significativamente diferentes $(\lambda 1=0.00006, P<$ $0.00005 ; \lambda 2=0.0004, P<0.00005$, lo que explica $86.3 \%$ de la variación acumulada), con 95.7 \% de casos correctamente clasificados.

Las variables más importantes para la primera función discriminante (FD I) están relacionadas principalmente con la precipitación: Bio 17 (precipitación del trimestre más seco), Bio 18 (precipitación del trimestre más cálido), además de Bio 3 (isotermalidad), Bio 4 (estacionalidad de la temperatura) y Bio 12 (precipitación anual); y para la segunda función discriminante (FD II) las variables de temperatura Bio 5 y Bio 7, que se refieren a la temperatura máxima del mes más cálido y a la oscilación anual de la temperatura, respectivamente.

En la gráfica obtenida con las dos primeras funciones discriminantes (Figura 3), se puede observar que los valores de la FD I separan dos grupos. El Grupo I formado por las especies F. haematacanthus, F. pilosus y F. histrix con requerimientos de precipitaciones promedio más altas (de 438.3 a $542.1 \mathrm{~mm}$ anuales), y estacionalidad de la temperatura menor (de 0.6 a $1.1 \%$ ), se localizan en el Altiplano Mexicano y el centro del país (Figura 1B).

El Grupo II está conformado por las especies restantes con precipitaciones promedio de 77.7 a $257.3 \mathrm{~mm}$ anuales 


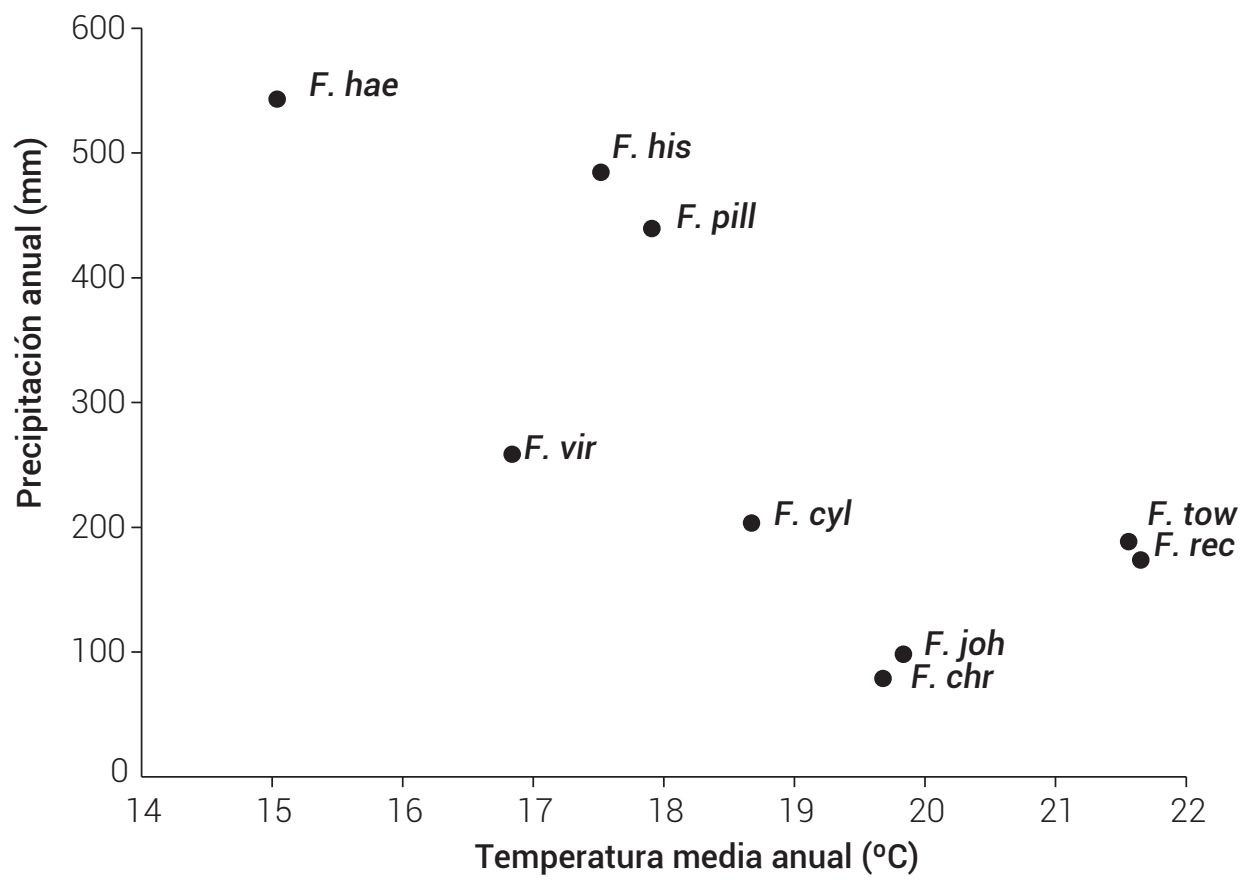

Figura 2. Relación de las variables ambientales temperatura media anual y precipitación de las especies de Ferocactus. $F$. hae: F. haematacanthus; F. his: F. histrix; F. pil.: F. pilosus; F. vir. F. viridescens; F. cyl: F. cylindraceus; F. tow.: F. townsendianus; F. rec.: F. rectispinus; F. joh.: F. johnstonianus; F. chr.: F. chrysacanthus.

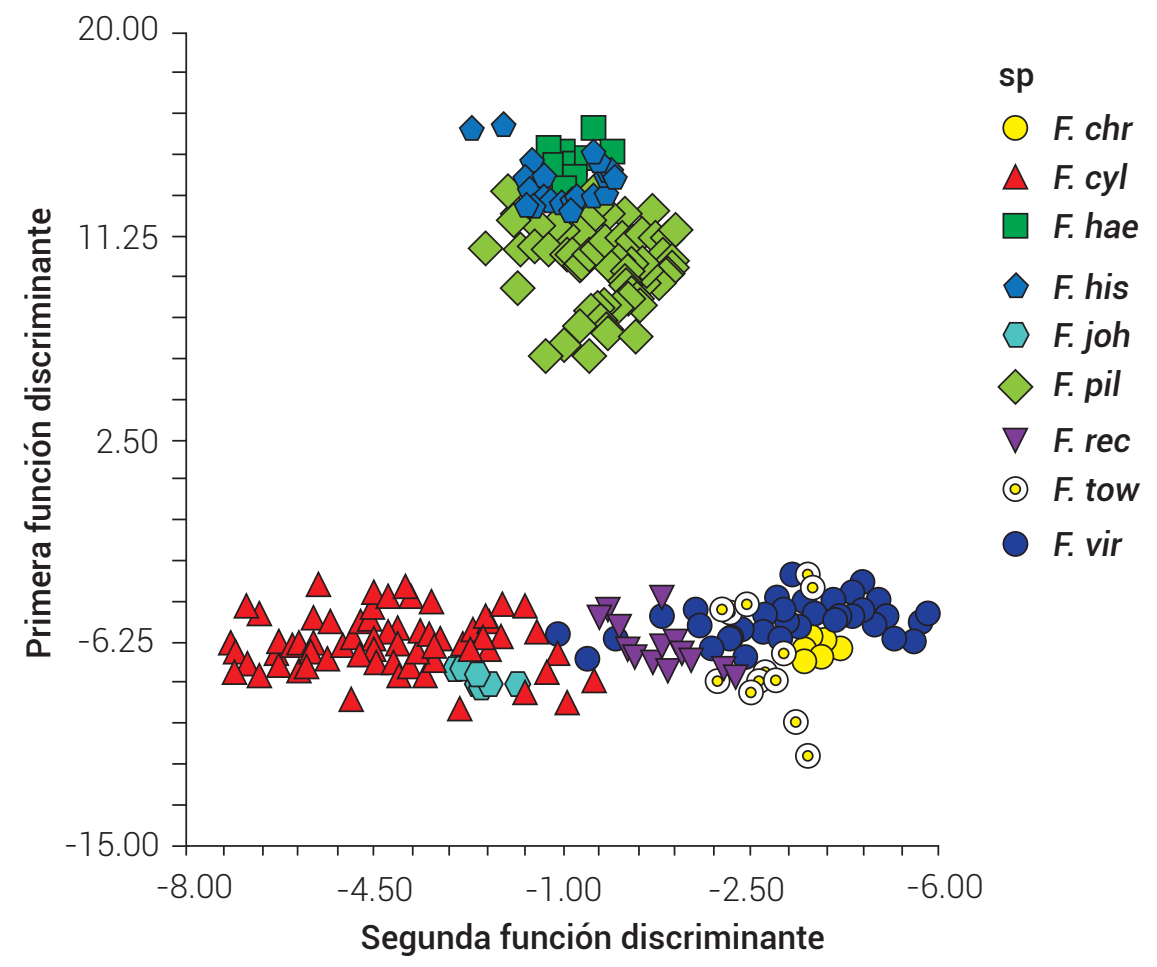

Figura 3. Análisis de función discriminantes con base en las variables climáticas (Cuadro 1) para las especies de Ferocactus. F. chr.: F. chrysacanthus; F. cyl: F. cylindraceus; F. hae: F. haematacanthus; F. his: F. histrix; F. joh.: F. johnstonianus; F. pil.: F. pilosus; F. rec.: F. rectispinus; F. tow.: F. townsendianus; F. vir. F. viridescens. 
y estacionalidad de la temperatura de 0.6 a $0.7 \%$, y se distribuyen en la Península de Baja California (Figura 1A). Este Grupo II está dividido a su vez por el FD II en el subgrupo ॥-1, que está formado por las especies de ambientes más secos F. cylindraceus y F. johnstonianus, localizadas en la costa oriental de Baja California (Figura 1A), y por el subgrupo II-2 que agrupa a F. rectispinus, F. towsendianus, F. viridescens y $F$. chrysacanthus, que se distribuyen desde Baja California y Baja California Sur por la costa occidental (Figura 1A, Cuadro 3). Este último grupo adquiere mayor importancia al contener todas las especies amenazadas, de acuerdo con la legislación ambiental federal mexicana.

Los resultados hacen referencia, por una parte, a que si bien las especies de Ferocactus están adaptadas a zonas semiáridas, cada especie tiene sus propias limitantes ambientales, y se distribuyen dentro de un gradiente de condiciones ambientales que van desde las zonas muy áridas y calurosas hasta las de aridez media y temperaturas menos extremosas. Así, la problemática de conservación se puede incrementar cuando estas especies que hasta ahora se encuentran amenazadas por las actividades antrópicas, también lo podrían estar por efectos del cambio climático global, donde las variaciones estimadas en la temperatura (incremento) y precipitación (disminución) (Arriaga y Gómez, 2004), podrían presentar efectos adversos en la distribución de las especies, cambios de abundancia, estructura y dinámica, fenológica y fisiológicamente, entre otros (Ferrier y Guisan, 2006), principalmente en las especies que muestren una distribución restringida ( $F$. chrysacanthus, F. haematacanthus y F. johnstonianus), o en aquellas que muestren una especificidad ambiental.

\section{CONCLUSIONES}

Las colecciones biológicas constituyen la fuente primaria para obtener información acerca de la biodiversidad de un área o bien para inferir la distribución de las especies. Gracias a los avances tecnológicos cada día el acceso y análisis de los datos biológicos se realizan en menor tiempo. En este sentido, los mapas de distribución producidos en este trabajo, presentan zonas en donde las especies posiblemente se encuentran, pero aún no han sido registradas. Además, el área de distribución potencial calculada, puede ser utilizada como criterio para analizar de nuevo las categorías de riesgo en las que se encuentran.

De la misma forma, se puede concluir que pese a los esfuerzos de garantizar la preservación y conservación de nuestro patrimonio natural por medio de Áreas Naturales Protegidas, éstas resultan aún insuficientes, ya que el análisis realizado muestra una baja representatividad del área de distribución de siete especies de Ferocactus. El presente estudio podría incrementar sus alcances en las ANP's para las especies analizadas, con el uso de recientes herramientas digitales (modelos de nicho ecológico y distribución potencial) que no han sido consideradas dentro de los programas de manejo de las mismas, lo que permitiría identificar zonas de mayor importancia dentro del ANP.

Por otra parte, es importante incrementar los estudios sobre los Ferocactus, en términos de aspectos biológicos, ecológicos, geográficos, ambientales, usos, entre otros, que permitan conocer ampliamente a las especies o contener información primordial. Actualmente se reconocen 10 especies clasificadas en alguna categoría de riesgo de acuerdo con la NOM-059-SEMARNAT-2010; sin embargo, es posible que esta lista pueda incrementarse o modificarse, ante los cambios ambientales globales presentes.

\section{AGRADECIMIENTOS}

Al Dr. Alejandro Zavala Hurtado por su amable revisión y comentarios al resumen, así como a los revisores anónimos por sus comentarios al contenido del manuscrito. Este trabajo forma parte del proyecto "Diversidad ambiental, diversidad biológica y cambio climático: implicaciones para la conservación" aprobado por la División de Ciencias Biológicas y de la Salud de la UAM-Iztapalapa.

\section{BIBLIOGRAFÍA}

Anderson E. F. (2001) The Cactus Family. Timber Press Inc. Portland, Oregon. $776 p$

Arias S., S. Gama-López, L. U. Guzmán-Cruz y B. Vázquez-Benítez (2012) Flora del Valle de Tehuacán-Cuicatlán. Fascículo 95. Cactaceae. Instituto de Biología, UNAM. México, D. F., México. 240 p.

Arias S., U. Guzmán, M. C. Mandujano, M. Soto G. y J. Golubov (2005) Las especies mexicanas de cactáceas en riesgo de extinción l. Una comparación entre los listados NOM-059-ECOL-2001 (México) La Lista Roja (UICN) y CITES. Cactáceas y Suculentas Mexicanas 50:100-125

Arriaga L. y L. Gómez (2004) Posibles efectos del cambio climático en algunos componentes de la biodiversidad de México. In: Cambio Climático: una Visión desde México. J. Martínez y A. Fernández B. (comps.). Secretaría de Medio Ambiente y Recursos Naturales. Instituto Nacional de Ecología. México. pp:255-265.

Challenger A. y R. Dirzo (2009) Tendencias de cambio y estado de la biodiversidad, los ecosistemas y sus servicios. In: Capital Natural de México. Vol. II: Estado de Conservación y Tendencias de Cambio. Comisión Nacional para el Conocimiento y Uso de la Biodiversidad. México. pp:37-73.

CITES, Convención sobre el Comercio Internacional de Especies Amenazadas de Fauna y Flora Silvestres (2017) Apéndices I, II y III en vigor a partir del 2 de enero de 2017. https://cites.org/sites/default/files/ esp/app/2017/S-Appendices-2017-01-02.pdf.

CONABIO, Comisión Nacional para el Conocimiento y Uso de la Biodiversidad (2016) Portal de Geoinformación, Sistema Nacional de Información sobre Biodiversidad. http://www.conabio.gob.mx/ informacion/gis/.

CONANP, Comisión Nacional de Áreas Naturales Protegidas (2012) Áreas Naturales Protegidas Federales de México. http://www. conabio.gob.mx/informacion/gis/

Contreras-Medina R., I. Luna-Vega y C. A. Ríos-Muñox (2010) Distribución de Taxus globosa (Taxaceae) en México: Modelos ecológicos de nicho, efectos del cambio del uso del suelo y conservación. Revista Chilena de Historia Natural 83:421-433.

Cota-Sánchez J. H. (2008) Evolución de cactáceas en la región del Golfo 
de California. In: Estudios de las Islas del Golfo de California. L. M. Flores-Campaña (ed.). Universidad Autónoma de Sinaloa Gobierno del Estado de Sinaloa-Consejo Nacional de Ciencia y Tecnología. México. pp:67-79.

Cruz-Cárdenas G., J. L. Villaseñor, L. López-Mata, E. Martínez-Meyer y E. Ortiz (2014) Selección de predictores ambientales para el modelado de la distribución de especies en MaxEnt. Revista Chapingo Serie Ciencias Forestales y del Ambiente 20:187-201. http:// dx.doi.org/10.5154/r.rchscfa.2013.09.034

Cuervo-Robayo A. P., O. Téllez-Valdés, M. A. Gómez-Albores, C. S. VenegasBarrera, J. Manjarrez and E. Martínez-Meyer (2013) An update of high-resolution monthly climate surfaces for Mexico. International Journal of Climatology 34:2427-2437, doi:10.1002/ joc.3848.

Delgadillo R. J. (2004a) Ferocactus johnstonianus. Actualización de las especies de plantas incluidas en la NOM-059-ECOL-2000. Herbario BCMEX, Facultad de Ciencias, Universidad Autónoma de Baja California. Bases de datos SNIB-CONABIO. Proyecto W037. México. D.F., http://www.conabio.gob.mx/conocimiento/ise/fichasnom/Ferocactusjohnstonianus00.pdf (Marzo 2015).

Delgadillo R. J. (2004b) Ferocactus viridescens. Actualización de las especies de plantas incluidas en la NOM-059-ECOL-2000. Herbario BCMEX, Facultad de Ciencias, Universidad Autónoma de Baja California. Bases de datos SNIB-CONABIO. Proyecto W037. México. D.F., http://www.conabio.gob.mx/conocimiento/ise/fichasnom/Ferocactusviridescens00.pdf (Marzo 2015).

DOF, Diario Oficial de la Federación (2010) Norma Oficial Mexicana NOM059-SEMARNAT-2010, Protección Ambiental-Especies Nativas de México de Flora y Fauna Silvestres-Categorías de Riesgo y Especificaciones para su Inclusión, Exclusión o Cambio-Lista de Especies en Riesgo. México. 78 p.

Elith J., C. H. Graham, R. P. Anderson, M. Dudík, S. Ferrier, A. Guisan, R. J. Hijmans, F. Huettmann, J. R. Leathwick, A. Lehmann, J. Li, L. G. Lohmann, B. A. Loiselle, G. Manion ,C. Moritz, M. Nakamura, Y. Nakazawa, J. McC. Overton, A. T. Peterson, S. J. Phillips, K. S. Richardson, R. ScachettiPereira, R. E. Schapire, J. Soberón, S. Williams, M. S. Wisz and N. E. Zimmermann (2006) Novel methods improve prediction of species' distributions from occurrence data. Ecography 29:129151.

Felicísimo Á. M., J. Muñoz, C. J. Villalba y R. G. Mateo (2011) Impactos, Vulnerabilidad y Adaptación al Cambio Climático de la Biodiversidad Española. 1 Flora y Vegetación. Oficina Española de Cambio Climático, Ministerio de Medio Ambiente, y Medio Rural y Marino. Madrid, España. 552 p.

Ferrier S. and A. Guisan (2006) Spatial modelling of biodiversity at the community level. Journal of Applied Ecology 43:393-404

GBIF, Global Biodiversity Information Facility (2016) Biodiversity data. GBIF. org (3rd March 2015) GBIF Occurrence Download. http://doi. org/10.15468/dl.jqfyrx (Marzo 2015).

Goettsch B., C. Hilton-Taylor, G. Cruz-Piñón, J. P. Duffy, A. Frances, H. M. Hernández, R. Inger, C. Pollock, J. Schipper, M. Superina, N. P. Taylor, M. Tognelli, A. M. Abba, S. Arias, H. J. Arreola-Nava, M. A. Baker, R. T. Bárcenas, D. Barrios, P. Braun, C. A. Butterworth, A. Búrquez, F. Caceres, M. Chazaro-Basañez, R. Corral-Díaz, M. Del Valle-Perea, P. H. Demaio, W. A. Duarte de Barros, R. Durán, L. Faúndez-Yancas, R. S. Felger, B. Fitz-Maurice, W. A. Fitz-Maurice, G. Gann, C. Gómez-Hinostrosa, L. R. Gonzales-Torres, M. P. Griffith, P. C. Guerrero, B. Hammel, K. D. Heil, J. G. Hernández-Oria, M. Hoffmann, M. Ishiki Ishihara, R. Kiesling, J. Larocca, J. L. León-de la Luz, C. R. Loaiza, M. Lowry, M. C. Machado, L. C. Majure, J. G. Martínez-Ávalos, C. Martorell, J. Maschinski, E. Méndez, R. A. Mittermeier, J. M. Nassar, V. Negrón-Ortiz, L. J. Oakley, P. OrtegaBaes, A. B. Pin- Ferreira, D. J. Pinkava, J. M. Porter, R. Puente-Martinez, J. R. Gamarra, P. Saldivia-Pérez, E. Sánchez- Martínez, M. Smith, J. M. Sotomayor, S. N. Stuart, J. L. Tapia-Muñoz, T. Terrazas, M. Terry, M. Trevisson, T. Valverde, T. R. Van Devender, M. E. Véliz-Pérez, H. E. Walter, S. A. Wyatt, D. Zappi, J. A. Zavala-Hurtado and K. J. Gaston (2015) High proportion of cactus species threatened with extinction. Nature Plants doi:10.1038/NPLANTS.2015.142.

Guzmán U., S. Arias y P. Dávila (2007) Catálogo de Cactáceas Mexicanas. Universidad Nacional Autónoma de México y Comisión Nacional para el Conocimiento y Uso de la Biodiversidad. México, D.F. $315 p$.
Hernández H. M. y H. Godínez A. (1994) Contribución al conocimiento de las cactáceas amenazadas. Acta Botánica Mexicana 26:33-52.

Hutchinson G. E. (1957) Concluding remarks. Cold Spring Harbor Symposium on Quantitative Biology 22:415-427.

INEGI, Instituto Nacional de Estadística y Geografía (2013) Conjunto de datos vectoriales de uso de suelo y vegetación. Escala 1:250 000. Serie V. 2da. Edición. Instituto Nacional de Estadística Geografía e Informática. Aguascalientes, México, http://www.conabio. gob.mx/informacion/gis/ (Febrero 2015).

INEGI, Instituto Nacional de Estadística y Geografía (2016) Anuario estadístico y geográfico de Baja California 2016. Instituto Nacional de Estadística y Geografía. México. 427 p.

IUCN, The IUCN Red List of Threatened Species (2016) [Internet], Version 2016-1, Gland, Switzerland, International Union for the Conservation of Nature. http://www.iucnredlist.org. (December 2016).

Jiménez-Sierra C. L. (2011) Las cactáceas mexicanas y los riesgos que enfrentan. Revista Digital Universitaria 12:3-22.

León de la Luz J. L. (2003a) Ferocactus chrysacanthus. Información sobre algunas especies de cactáceas incluidas en la Norma Oficial Mexicana de Baja California Sur. Centro de Investigaciones Biológicas del Noroeste SC. Bases de datos SNIB-CONABIO. Proyecto W014. México. D.F.

León de la Luz J. L. (2003b) Ferocactus rectispinus. Información sobre algunas especies de cactáceas incluidas en la Norma Oficial Mexicana de Baja California Sur. Centro de Investigaciones Biológicas del Noroeste SC. Bases de datos SNIB-CONABIO. Proyecto W014. México. D.F.

León de la Luz J. L. (2003c) Ferocactus townsendianus townsendianus. Información sobre algunas especies de cactáceas incluidas en la Norma Oficial Mexicana de Baja California Sur. Centro de Investigaciones Biológicas del Noroeste SC. Bases de datos SNIB-CONABIO. Proyecto W014. México. D.F.

Londoño-Murcia M. C. y V. Sánchez-Cordero (2011) Distribución y conservación de especies amenazadas en Mesoamérica, Chocó y Andes tropicales. Revista Mexicana de Biodiversidad 82:926-950.

Meza N. V. (2011) Cactáceas Mexicanas: Usos y Amenazas. Segundo informe referente a la realización de la asesoría número INE/ ADA-026/2011. México. 1209 p.

Peterson A. T., M. A. Ortega-Huerta, J. Bartley, V. Sánchez-Cordero, J. Soberón, R. H. Buddemeier and D. R. B. Stockwell (2002) Future projections for Mexican faunas under global climate change scenarios. $\mathrm{Na}$ ture 416:626-629.

Phillips S. J., R. P. Anderson and R. E. Schapire (2006) Maximum entropy modeling of species geographic distributions. Ecological Modelling 190:231-259.

Pilbeam J. and D. Bowdery (2005) Ferocactus. British Cactus \& Succulent Society. Norwich, England. $116 \mathrm{p}$.

Raxworthy C. J., E. Martínez-Meyer, N. Horning, R. A. Nussbaum, G. E. Schneider, M. A. Ortega-Huerta and A. T. Peterson (2003) Predicting distributions of known and unknown reptile species in Madagascar. Nature 426:837-841.

REMIB, Red Mundial de Información sobre Biodiversidad (2015) http://www. conabio.gob.mx/remib/doctos/remib_esp.html (Marzo 2015).

Sánchez-Cordero V., P. Illoldi-Rangel, M. Linaje, S. Sarkar and A. T. Peterson (2005) Deforestation and extant distributions of Mexican endemic mammals. Biological Conservation 126:465-473.

Soberón J. and A. T. Peterson (2005) Interpretation of models of fundamental ecological niches and specie's distributional areas. Biodiversity Informatics 2:1-10.

StatSoft (2010) Statistica 9.1. StatSoft Inc.: Tulsa, OK, USA

UNIBIO, Unidad de Informática para la Biodiversidad. MEXU Consulta de ejemplares del Herbario Nacional. http://unibio.unam.mx/minero/index.jsp?accion=sc\&colecciones $=M E X U$, Herbario (Enero 2015).

U. S. Geological Survey (2015) HYDRO1K. https://lta.cr.usgs.gov/HYDR01K/ (Marzo 2015).

Vázquez-Sánchez M., T. Terrazas, S. Arias and H. Ochoterena (2013) Molecular phylogeny, origin, and taxonomic implications of tribe Cacteae (Cactaceae). Systematics and Biodiversity 11:103-116.

Villaseñor J. L. y 0. Téllez-Valdés (2004) Distribución potencial de especies del género Jefea (Asteraceae) en México. Anales del Instituto de Biología. Serie Botánica 75:205-220. 